\title{
DeSERCión Escolar y TRABajo JuVEniL: ¿Dos Caras de Una Misma Decisión?*
}

\author{
Claudio Sapelli \\ Arístides TORCHE \\ Pontificia Universidad Católica de Chile
}

\begin{abstract}
En Chile, prácticamente no hay deserción en educación básica; sin embargo, en secundaria la tasa de deserción es de un 10\%. Este artículo estudia los determinantes de dicha tasa de deserción. Para ello presenta un modelo microeconométrico de elección bivariada que permite estudiar conjuntamente dos decisiones diferentes, pero relacionadas: la de deserción escolar y la de participar en la fuerza laboral. Estas decisiones pueden considerarse relacionadas ya que muchos de los que desertan lo hacen para trabajar, pero son diferentes, puesto que no todos los jóvenes que desertan van al mercado laboral, algunos permanecen en sus hogares, o trabajan en la familia y no son remunerados. El estudio encuentra que la educación de los padres tiene impacto significativo en ambas decisiones y lo mismo sucede con las variables de caracterización demográfica de los hogares. Finalmente, se obtiene un efecto del ingreso en la decisión de desertar que si bien es estadísticamente significativo, es cuantitativamente pequeño, lo que cuestiona la efectividad de algunas de las politicas públicas utilizadas para resolver el problema de la deserción.
\end{abstract}

JEL: I1, D1, J2

Palabras clave: Educación, Deserción Escolar, Participación Mercado Laboral.

\section{INTRODUCCIÓN}

En Chile, prácticamente todos los niños asisten a la escuela hasta la edad de 13 años. Sin embargo, de allí en adelante comienza un proceso de deserción que es el objeto de estudio de este trabajo. Se estudiará el grupo de edad de 14 a 17 años, que debiera asistir a la educación media. En dichas edades un 10\% de la población no asiste a la escuela (dicho porcentaje va de $4 \%$ a los 14 años a $16 \%$ a los 17 años). ${ }^{1}$ El estudio puede dar luz sobre si serían necesarias medidas de

\footnotetext{
* Los autores agradecen la valiosa ayuda de Catalina Braun y los comentarios de dos árbitros anónimos, y de Bernardita Vial, José Miguel Sánchez y Ricardo Paredes.

${ }^{1}$ Todos los datos son de la encuesta CASEN 1996, que fue utilizada para el trabajo empírico.
} 
política que incentiven la permanencia escolar, y cuáles podrían ser las más efectivas.

Una explicación para el aumento de la deserción en esas edades puede encontrarse en la mayor posibilidad de trabajo de los niños, por su mayor edad y nivel de educación. Aquí estudiaremos las decisiones de desertar o asistir, y la de participar en la fuerza laboral conjuntamente, en parte con la intención de determinar si son como dos caras de un mismo proceso de decisión. Los jóvenes que desertan del sistema educacional no sólo tienen como opción trabajar en una empresa, sino también hacerlo en la casa, o permanecer inactivos. En efecto, sólo un 44\% de los que desertaron en 1996 se incorporaron a la fuerza de trabajo.

Cuando la deserción se asocia a la incorporación a la fuerza de trabajo, esta decisión presenta beneficios inmediatos por el dinero ganado en el trabajo y beneficios de mediano plazo, que surgen de la más temprana experiencia laboral; pero también presenta efectos negativos de mediano y largo plazo, que surgen de un abandono temprano de la enseñanza formal, en términos de menor capital humano y posiblemente menores ingresos en una perspectiva más permanente. ${ }^{2}$ En este sentido, personas que se encuentran presionadas por la coyuntura pueden tomar decisiones inconvenientes del punto de vista del mediano y largo plazo (en otras palabras, actúan como si su tasa de descuento fuese mayor a la social).

Los antecedentes disponibles muestran que para el año 1996, del total de jóvenes en el grupo de edad 14 -17 años, un 87,7\% asistía a establecimientos de educación media y no trabajaba en el mercado laboral; un 4,5\% trabajaba y no asistía; un 5,7\% ni trabajaba en el mercado ni asistía, y un 2,1\% trabajaba y asistía.

Entre las hipótesis que se considerarán respecto de los determinantes de la decisión de deserción escolar, está una manejada con frecuencia en la discusión de política: que la situación económica de la familia hace necesario que los jóvenes trabajen. Sin embargo, el ingreso monetario de un empleo no es necesariamente la única razón para desertar, puesto que, como se señaló anteriormente, casi un $6 \%$ no asiste a la escuela ni está trabajando. A su vez, como se verá en la revisión de la literatura, muchos trabajos empíricos encuentran que los cambios en el ingreso tienen efectos marginales muy pequeños sobre las decisiones de desertar y trabajar. $^{3}$

${ }^{2}$ La razón por la cual se utilizó la expresión "posiblemente menores ingresos en una perspectiva más permanente" se refiere al hecho de que es necesario estudiar si las personas que abandonan la educación media son lo suficientemente similares a aquellas que la continúan, para que uno pueda atribuirles la tasa de retorno de estos últimos a los primeros. En general, uno debiera esperar que esto no fuera así (ver Willis y Rosen, 1979 o Eckstein y Wolpin, 1999). Es decir, aquellos que se educan menos lo hacen, porque tienen ventajas comparativas en adquirir experiencia laboral y desventajas comparativas en acumular capital humano vía educación formal.

${ }^{3}$ Conviene mencionar brevemente que entre dichas condiciones es necesario tomar en cuenta las regulaciones del mercado laboral, tal como los cambios en el salario mínimo. Este factor ha concitado el interés por parte del reducido número de estudios económicos existente en Chile respecto a la decisión de desertar. Se postula que el aumento del salario mínimo tendría un efecto de incrementar la deserción, aunque los trabajos empíricos no son concluyentes al respecto. El salario mínimo no se tendrá en cuenta en este trabajo. Véase Paredes y Sanhueza (1996) y Bravo y Contreras (2000). 


\section{La Deserción y El Trabajo Infantil en La Literatura ECONÓMICA}

Hay una amplia literatura empírica, mucha de ella reciente, que analiza el tema de este trabajo, la mayor parte de las veces con el objetivo de encontrar formas de evaluar las medidas de política para disminuir la deserción. Sólo una parte de la literatura estudia conjuntamente las decisiones de desertar y trabajar, como lo hace este trabajo. Hay varias sorpresas que han aparecido en la literatura empírica, en particular, si se las compara con las preconcepciones que existían. Por ejemplo, el ingreso no es tan importante como se creía. De hecho, trabajos recientes como el de Ravallion y Woodon (2000) argumentan que hay poca evidencia a favor de la idea de la trampa de la pobreza, de lo que se concluye que el trabajo infantil no es una fuerza importante en perpetuar la pobreza.

Otro factor que ha influido en esta última conclusión es que se ha establecido que las políticas que han sido exitosas en aumentar la asistencia no necesariamente disminuyen el trabajo infantil. Por ejemplo, Ravallion y Woodon (2000) obtienen que estas estrategias no disminuyen el trabajo infantil en Bangladesh, en tanto que Skoufias y Parker (2001) encuentran que sí lo hacen en México. Es claro que siempre existe la posibilidad de trabajar a tiempo parcial y estudiar, sacrificando ocio. Por eso no es necesariamente cierto que la decisión de asistir y de trabajar sean imágenes espejo una de la otra.

La opción de asistir a la escuela versus la de no hacerlo es analizada por Gertler y Glewwe (1992). Los autores encuentran que los padres, en las zonas rurales de Perú, asignan mayor valor a la asistencia a la escuela de los niños hombres que la de las niñas. Para investigar este hecho utilizan un modelo logit multinomial anidado. Entre los resultados más destacables encontramos que la demanda por educación aumenta con el nivel de gasto del hogar (elasticidad ingreso positiva); los padres demandan menos educación para las hijas mujeres (hay discriminación por su mayor salario de reserva en el hogar); la educación de los padres tiene un efecto menor en la demanda por educación (en particular comparado con el de la madre), y las variables que reflejan calidad (tiempo de viaje y falta de profesores) tienen un efecto negativo.

Skyt (1998) estudia la asistencia a la escuela y la participación en la fuerza de trabajo de menores de entre 7 y 14 años en Zambia. Para ello emplea un modelo probit bivariado que permite analizar el efecto de las variables consideradas en las decisiones: solamente estudiar, solamente trabajar, trabajar y estudiar y no trabajar ni estudiar. Es esta versatilidad de análisis de las diferentes alternativas de decisión posibles, lo que proporciona su mayor interés a este modelo que servirá de base al que se emplea en este trabajo.

El modelo de Skyt es estimado en dos etapas por la existencia de regresores endógenos: primero se estima por MICO cada uno de los regresores endógenos con variables instrumentales y luego, se realiza un probit, incluyendo las variables endógenas y los residuos de mínimos cuadrados de la etapa anterior.

Los resultados más destacables son: que los no observables, que influyen negativamente sobre la decisión de asistir al colegio, lo hacen positivamente sobre 
la decisión de trabajar; las variables que miden calidad en general tienen el signo opuesto al esperado; el efecto del ingreso es muy pequeño, por lo que bajos ingresos y pobreza no son las principales explicaciones del trabajo; y, finalmente, que las variables más importantes son la tradición de la familia y el acceso al colegio.

Usando datos de Chile, Beyer (1998) estima dos modelos logit, uno para explicar la probabilidad de estar desempleado (dado que uno está en la fuerza de trabajo); y un segundo para explicar la probabilidad que un joven de 15 a 24 esté estudiando. En este último logit incluye las variables edad, sexo, educación de los padres, situación familiar (un padre o dos), localización (urbano o rural), situación civil (casado o no) y el ingreso per cápita del hogar. El centro de atención del trabajo es la desocupación juvenil, y este logit cumple el propósito de proveer evidencia a una posible solución de la misma, vía subsidios a la educación (reteniendo a los jóvenes en la escuela). El trabajo no cuenta con un marco teórico formal, y atribuye el retorno de los egresados a sus diferencias con los no egresados (no considera la posibilidad de que haya un sesgo de selección). Los resultados obtenidos proveen la evidencia de que entre los desempleados hay jóvenes que están voluntariamente desempleados a la espera de una oferta laboral más adecuada a sus pretensiones, lo cual podría explicar la alta tasa observada de inactividad. También se presenta evidencia preliminar de que mientras más bajo sea el ingreso de la familia, más alta es la probabilidad de que un joven deje de estudiar. Por esto, concluye que no deben descartarse las políticas sociales que reduzcan la deserción en dichos sectores (como el subsidio educacional).

Eckstein y Wolpin (1999) estiman un modelo de decisión secuencial de asistencia a la enseñanza media y trabajo. Se supone que los jóvenes eligen entre combinaciones de trabajo/escuela en orden a maximizar el valor presente de la utilidad esperada en cada período. Los resultados obtenidos indican que los que desertan tienen rasgos distintos a los que se gradúan: menor habilidad para el colegio o menor motivación, menores expectativas de los beneficios de graduarse, ventajas comparativas en trabajos que realizan los "no graduados", mayor valor del ocio, menor valor de consumo de asistir al colegio. Se constata, además, que las legislaciones que prohíben el trabajo tienen poco impacto en las tasas de graduación; y que para disminuir la deserción en educación media hay que afectar la habilidad y la motivación, y también los rasgos iniciales, como el valor esperado de graduarse.

Ravallion y Wodon (1999) testean la sustitución que existe entre trabajo infantil y asistencia al colegio. Se analizan los efectos sobre ambos de un subsidio Food-For-Education (FFE) dado por asistencia en Bangladesh. Se estiman dos probit bivariados, uno para trabajo y otro para asistencia al colegio. Los resultados que se obtienen son: el subsidio tiene un efecto negativo en la participación en la fuerza laboral y un efecto positivo en la probabilidad de asistir al colegio; la disminución del trabajo es menor que lo que se gana en aumento de escolaridad (es sólo un cuarto del aumento en asistencia para los hombres y un octavo para las mujeres); la educación de los padres tiene un efecto muy significativo; las variables demográficas de la familia tienen un efecto débil; no existe mucho apoyo 
empírico a la idea de la trampa o círculo de la pobreza; y se concluye que los padres sustituyen otros usos del tiempo de los hijos para aumentar la escolaridad, y así el trabajo disminuye en menor grado de lo esperado.

Margo y Finegan (1993) corren regresiones para EE.UU. estimadas por mínimos cuadrados y mínimos cuadrados en dos etapas (series de tiempo). Los resultados ponen de manifiesto que la demanda por educación de los padres es uno de los principales factores detrás de la tendencia de ir aumentando la escolaridad de los hijos y eso explica en gran medida la caída en la fuerza laboral de los jóvenes; y no sólo son causales el proceso de industrialización y el salario mínimo como se pensaba anteriormente.

Ranjan (2000) utiliza un procedimiento en dos etapas para estimar ecuaciones de oferta de trabajo luego de corregir por sesgo de selección. Se estiman dos ecuaciones de forma reducida, una para el trabajo infantil y otra para la asistencia al colegio. Se analiza la hipótesis de que existe una asociación positiva entre horas de trabajo infantil y pobreza, y una asociación negativa entre asistencia al colegio y pobreza. Se utilizan datos de trabajo infantil y asistencia al colegio para niños en Perú (entre 6 y 17 años) y en Pakistán (entre 10 y 17 años). Ambas hipótesis se confirman para el caso de Pakistán, pero no para Perú. En todas las estimaciones, las horas de trabajo infantil responden positivamente a los salarios que reciben los niños. La mayor educación de los padres tiene un efecto positivo en la escolaridad de los niños.

Canagarajah y Coulombe (1998), al igual que Skyt, estiman modelos probit bivariados (variando las especificaciones y las variables) para analizar los determinantes del trabajo infantil en conjunto con la decisión de asistir al colegio, para niños entre 7 y 14 años en Ghana. Son modelos de forma reducida que mezclan variables del lado de la oferta y la demanda. Muestran que aumentar la demanda por educación es la manera más efectiva de disminuir el trabajo de los menores. A su vez, la educación del padre tiene un efecto negativo en el trabajo infantil, especialmente en los niños varones. La educación de la madre sólo influye en la participación en el colegio. La religión tiene un rol importante: los cristianos tienen una mayor asistencia al colegio, y luego los musulmanes. En los quintiles de ingresos más altos aumenta la asistencia. Se esperaba que el índice de bienestar (pobreza) tuviera una relación negativa con el trabajo; sin embargo, la relación no es lineal. Se encuentra una relación en forma de U invertida, particularmente en las zonas rurales. Se prueba que la pobreza no es el principal determinante del trabajo; $\mathrm{y}$, finalmente, las variables de presencia de los padres, edad y sexo de los hermanos no son significativas en el trabajo, pero sí la probabilidad de asistir al colegio aumenta si hay hermanas o gente mayor en el hogar.

El Cuadro 1 presenta un resumen del efecto de las principales variables consideradas en los diferentes trabajos. Puede observarse que en general los resultados empíricos validan las presunciones sobre las variables relacionadas con la permanencia en la escuela como ingreso, sexo, educación de los padres y otras variables demográficas de la familia. Sin embargo, su impacto no es sistemático. 


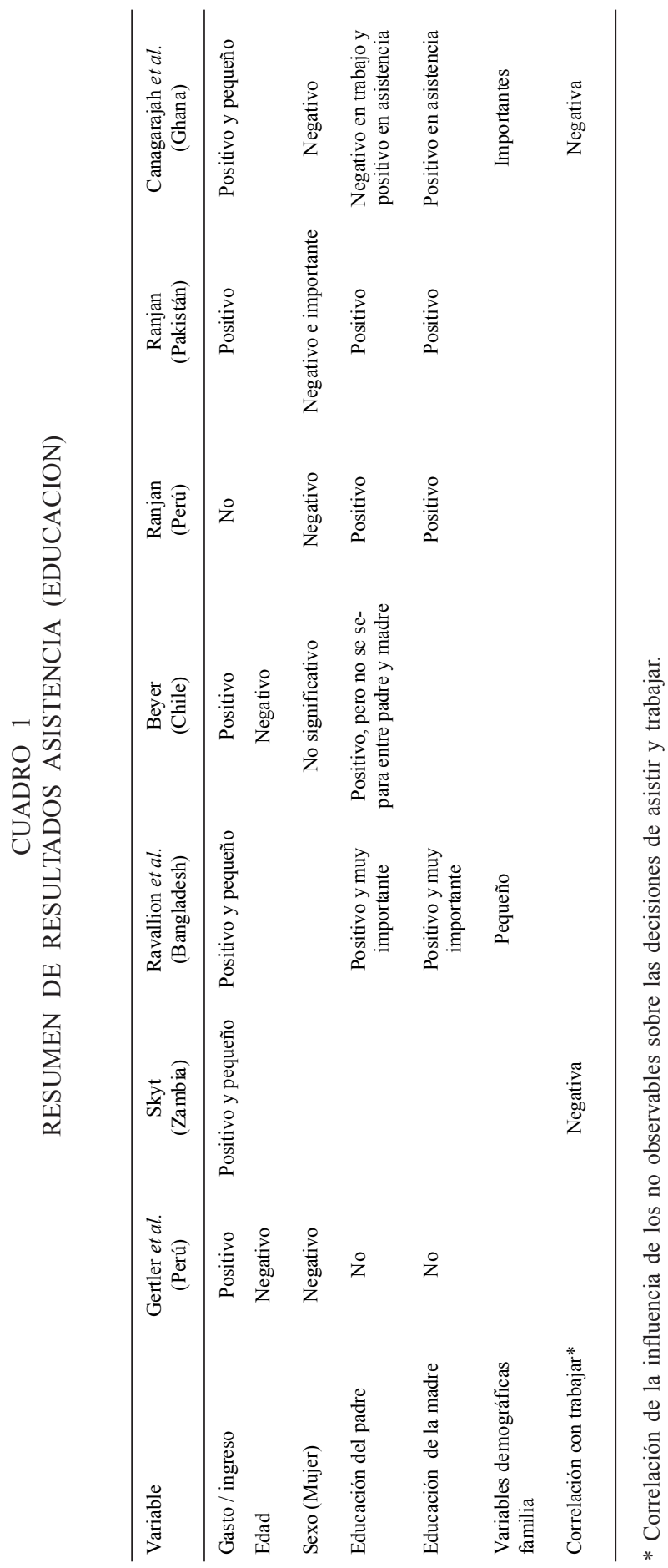


Como puede constatarse de la discusión bibliográfica, surgen varios hechos aceptados y numerosas dudas respecto a los determinantes de la decisión de desertar. Por ejemplo, se observa que a menor ingreso existe mayor probabilidad que el adolescente deba trabajar y, por lo tanto, mayor deserción. Sin embargo, este no es un resultado sistemático. Gertler y Glewwe (1992), Beyer (1998) y Ranjan (2000) (para Pakistán) encuentran un efecto positivo e importante del ingreso en la decisión de asistir. Sin embargo, Skyt (1998), Canagarajah y Coulombe (1998), Ravallion y Wodon (2000) y Ranjan (2000) (para Perú) encuentran que este efecto es pequeño. Como se verá, puede concluirse que el resultado es sensible a la metodología utilizada.

Respecto a la importancia del sexo del niño también se encuentran resultados muy diferentes, en particular en términos de magnitud. Las variables demográficas del hogar inciden según algunos estudios (Canagarajah y Coulombe) y no inciden según otros (Ravallion y Wodon).

Los trabajos analizados validan, en general, la presunción de que la educación de la madre es más importante en la decisión de asistir o desertar que el efecto de la educación del padre, que es de segundo orden. Sin embargo, Gertler y Glewwe encuentran que la educación de los padres no es importante. El resto de los trabajos revisados hallan un efecto positivo de la educación de los padres (de la madre como del padre) en la asistencia a la escuela de sus hijos. Canagarajah, de hecho, encuentra que no sólo hay un efecto positivo importante de la educación del padre en la decisión de asistencia, sino que la educación del padre tiene un efecto negativo importante en la decisión de trabajar (en tanto que la educación de la madre no es significativa en esta decisión).

Es interesante destacar otras vertientes que ha tomado la literatura sobre el tema. El trabajo de los niños puede ser considerado parte de un modelo de equilibrio más amplio en que su salario y nivel de contratación se determinan conjuntamente con el salario y contratación de los adultos e influyen, además, en las decisiones sobre las tasas de fertilidad de estos. Así, K. Basu y P. H. Van (1998) presentan un modelo del mercado de trabajo de los menores que se vincula con las características de fertilidad de los hogares considerada endógenamente. Dicho modelo se caracteriza por la existencia de múltiples equilibrios. Uno con alta fertilidad y trabajo de los menores y otro con ausencia de trabajo de niños en un ambiente de reducida fertilidad. Por otra parte, Angrist y Evans (1998) estudian las relaciones entre el tamaño de la familia y la oferta de trabajo de las mujeres. Para ello emplean un modelo con variables instrumentales en que el instrumento es el interés por la variedad en el sexo de los hijos. Así cuando los dos primeros hijos son del mismo sexo habría interés por aumentar la familia buscando el hijo del otro sexo, lo que en definitiva reduciría la probabilidad de oferta de trabajo femenina. Finalmente, Swinnerton y Rogers (1999), en un comentario al trabajo de Basu y P. H. Van, extienden el modelo de esos autores e incorporan la variable distribución de ingreso como otro factor de reducción del trabajo de los niños. Basu y P. H. Van (1999), en una réplica al comentario de Swinnerton y Rogers, ponen en perspectiva los resultados de dicho artículo distinguiendo el caso de los países más pobres (con ingreso per cápita inferior a los US\$1.000) en los que sería válida dicha propo- 
sición, de aquellos de ingreso per cápita entre US\$1.000 y US\$4.000 en que surge como una posible explicación adicional para el trabajo de los niños.

Sin negar la importancia de estas otras vertientes que ha enfatizado la literatura sobre la economía del trabajo de los niños, en este artículo se han considerado la fertilidad y la distribución del ingreso como variables exógenas y nos hemos concentrado en el vínculo entre el trabajo de los menores y el acervo de capital humano que se logra con más años de escolaridad, como factores generadores de beneficios al momento de tomar al decisión de continuar en la escuela o entrar a trabajar.

\section{La Asistencia a la Escuela, la Condición de InACtividad y suS CAUSAS}

El Cuadro 2 presenta los antecedentes de la población de jóvenes de entre 14 y 17 años clasificada por asistencia a un establecimiento educacional y por participación en la actividad productiva.

Visto desde el punto de vista del sistema educativo, los datos nos dicen que del total de jóvenes entre 14 y 17 años de edad, el 89,8\% asiste a un establecimiento educacional y el $10,2 \%$ no asiste. Si se calculan los porcentajes por filas, puede observarse que de los que asisten sólo el 2,3\% trabaja, de los que no asisten un $44,1 \%$ trabaja en el mercado. Existe, por lo tanto, una tercera categoría de análisis relevante, la inactividad (no asiste y no trabaja), que puede asociarse a trabajo en la casa o no, y que incluye al 55,9\% de los desertores .

Desde el punto de vista del mercado de trabajo (\% por columnas) de la población de 14 a 17, el 93,4\% no trabaja y el 6,6\% trabaja. De aquellos que no trabajan, un 93,9\% asiste a la educación media. De los que sí trabajan, un 31,8\% concurre a la educación media.

\section{CUADRO 2}

PORCENTAJES DE LA POBLACION QUE ASISTE A LAS ESCUELAS O QUE TRABAJA

\begin{tabular}{|c|c|c|c|c|c|c|c|c|}
\hline \multirow[b]{2}{*}{ ESCUELA } & \multicolumn{3}{|c|}{$\begin{array}{l}\text { TRABAJO } \\
\% \text { sobre total }\end{array}$} & \multicolumn{3}{|c|}{$\begin{array}{l}\text { TRABAJO } \\
\% \text { sobre filas }\end{array}$} & \multicolumn{2}{|c|}{$\begin{array}{c}\text { TRABAJO } \\
\% \text { sobre columnas } \\
\end{array}$} \\
\hline & No trabaja & Trabaja & Total & No trabaja & Trabaja & Total & No trabaja & Trabaja \\
\hline No asiste & 5,7 & 4,5 & 10,2 & 55,9 & 44,1 & 100,0 & 6,1 & 68,2 \\
\hline Asiste & 87,7 & 2,1 & 89,8 & 97,7 & 2,3 & 100,0 & 93,9 & 31,8 \\
\hline TOTAL & 93,4 & 6,6 & 100,0 & & & & 100,0 & 100,0 \\
\hline
\end{tabular}

Fuente: Cálculo de los autores sobre información de encuesta CASEN 1996.

Los resultados anteriores muestran que asistir y trabajar son dos decisiones diferentes, aunque relacionadas. Son relacionadas, porque el 94\% de los que 
no trabajan asiste y el $68 \%$ de los que trabajan no asiste. Pero son diferentes, porque no puede considerarse que no asistir sea simplemente la consecuencia de haber decidido trabajar, puesto que $56 \%$ de los que no asisten, tampoco trabaja. Por otra parte, sí es cierto que de los que asisten a establecimiento escolar, un 98\% no está trabajando.

En síntesis, en este artículo, asistir y trabajar serán consideradas dos decisiones diferentes, pero relacionadas y se intentará medir el nivel de correlación entre ellas.

Al estudiar las decisiones de asistencia y trabajo por edad, se observa que la asistencia se reduce de un $96 \%$ a los 14 años a un $84 \%$ a los 17 años. Es interesante observar, además, que la proporción de los que no asisten ni trabajan sube del $2 \%$ al $8 \%$ en dicho grupo etario, lo que muestra el impacto de otras razones fuera del trabajo que explicarían la deserción escolar.

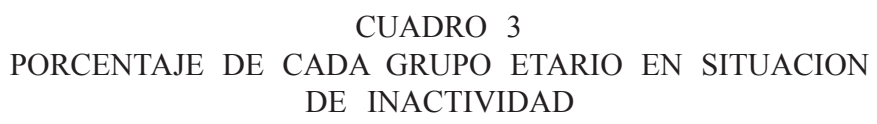

\begin{tabular}{ccccc}
\hline Edad & Urbano & Rural & Hombres & Mujeres \\
\hline 14 & 1,6 & 6,0 & 1,6 & 3,0 \\
15 & 4,2 & 8,6 & 4,8 & 5,1 \\
16 & 5,5 & 15,5 & 5,1 & 9,3 \\
17 & 7,1 & 16,1 & 4,1 & 12,9 \\
Total & 4,6 & 11,5 & 3,9 & 7,5 \\
\hline
\end{tabular}

Fuente: Cálculo de los autores sobre información de encuesta CASEN 1996.

El Cuadro 3 nuestra que para cualquier edad la proporción de desertores entre las mujeres es siempre superior a la de los hombres; que la de desertores en zonas rurales es superior a la de las zonas urbanas, y finalmente que la tasa de deserción aumenta con la edad, con la única excepción de lo que sucede con los hombres al pasar de 16 a 17 años. En todos estos casos es posible suponer que lo que desencadena dichas diferencias es un incremento en el costo alternativo de la ayuda en su hogar por parte de los jóvenes al pasar de zonas urbanas a rurales por la menor disponibilidad de equipamiento del hogar en estas últimas. En el caso de las mujeres en relación a los hombres, por la más directa relación que habitualmente han tenido las mujeres con las actividades cotidianas de una casa y finalmente por una mayor capacidad de desarrollar actividades en el hogar, incluyendo el cuidado de los menores, al aumentar la edad.

En términos agregados, las causas más importantes de la deserción son el hecho de estar trabajando, las dificultades económicas, ayudar en casa y la falta de interés. Los problemas económicos y de conducta adquieren más importancia en las primeras edades (14 y 15 años), en tanto que el trabajo es más importante 
entre los 16 y 17 años. El hecho de ayudar en casa mantiene una proporción constante en prácticamente todas las edades. ${ }^{4}$ (Véase Cuadro 4 ).

El Cuadro 4 muestra la posible importancia de ciertas variables como determinantes de la deserción. Entre ellas caben destacar la edad, la oferta de colegios, alguna medida de logro de los estudiantes para hacerse cargo de los problemas de conducta y repitencia, variables de riqueza y de necesidades en el hogar (que explican la demanda por trabajo en el hogar).

CUADRO 4
DESERCION ESCOLAR POR CAUSAS Y POR EDAD

\begin{tabular}{lrrrr}
\hline No asiste porque: & \multicolumn{1}{c}{14} & 15 & 16 & 17 \\
\hline Dificultad económica & 31,7 & 19,7 & 14,8 & 15,4 \\
Está trabajando & 13,4 & 15,3 & 24,1 & 27,1 \\
Ayuda en la casa & 10,1 & 5,4 & 11,7 & 10,4 \\
Maternidad o embarazo & 1,0 & 3,0 & 5,1 & 14,7 \\
No le interesa & 9,3 & 14,8 & 11,7 & 12,1 \\
Problemas de conducta & 11,9 & 17,4 & 10,8 & 4,0 \\
Está buscando trabajo & 0,4 & 3,8 & 3,4 & 7,8 \\
Otras & 22,2 & 20,6 & 18,4 & 18,5 \\
Total & 100,0 & 100,0 & 100,0 & 100,0 \\
\hline
\end{tabular}

Fuente: Cálculo de los autores sobre información de encuesta CASEN 1996.

\section{Las Decisiones de Estudiar, Trabajar o Permanecer Inactivo, y LAS VARIABLES CONSIDERADAS}

Desde el punto de vista metodológico, las decisiones de interés pueden ser evaluadas sobre la base de modelos de elección discreta o de varias alternativas, que permiten relacionar la probabilidad de elegir una de las alternativas planteadas, en este caso, seguir estudiando o no, con ciertas características del tomador de decisiones (la familia), del medio social en que se desenvuelve, de los establecimientos educacionales y de los propios educandos. Separadamente, se puede considerar la decisión de trabajar o no y estudiar su relación con las mismas características anteriores o con otras.

En este artículo se ha considerado que estudio y trabajo pueden ser modelados en términos de dos decisiones diferentes, aunque relacionadas: la de continuar estudiando versus no hacerlo y la de trabajar para mercado versus no hacerlo. Este esquema da origen a una matriz con cuatro resultados posibles que inclu-

\footnotetext{
${ }^{4} \mathrm{Al}$ observar las causas de inasistencia a un establecimiento educacional se pudo constatar que algunas de ellas correspondían a situaciones en que no es posible tomar ninguna de las decisiones en estudio. Estas se asocian a que la persona no puede trabajar ni estudiar por motivos de enfermedad o invalidez; porque están cumpliendo actividades como el servicio militar, y, finalmente, las personas que están preparando su PAA, puesto que ellas han terminado su ciclo medio de estudio. Estas categorías fueron excluidas del análisis.
} 
ye a los que estudian o trabajan solamente, a los que trabajan y estudian, y a los que no estudian ni trabajan.

Un elemento interesante de esta forma de analizar los datos es que la alternativa de no trabajar ni estudiar adquiere plena validez y, por lo tanto, puede ser investigada en términos de los factores concomitantes con ella. Para analizar estas dos decisiones, se emplea un modelo econométrico del tipo probit bivariado.

El modelo probit bivariado de función de índice con ecuaciones aparentemente no relacionadas, que es el que usaremos, supone la existencia de dos variables dicotómicas $y_{1}$ e $y_{2}$ que se interpretan como escolaridad y trabajo respectivamente. Dichas variables se asocian a otras tantas variables índices. $I_{1}$ y $I_{2}$ tal que $y_{1}=1$ si $I_{1}>0$ e $y_{2}=1$ si $I_{2}>0$. Si las variables $I$ toman valores no positivos entonces las $y$ son nulas. Las funciones $I_{1}$ e $I_{2}$ pueden ser descritas por un conjunto de variables de decisión $\mathrm{X}_{1} \mathrm{y} \mathrm{X}_{2}$ más sendas variables aleatoria $\varepsilon_{1} \varepsilon_{2}$, que en general no son independientes entre sí. En términos formales se tiene que

$$
\begin{aligned}
& y_{1}=1 \text { si } I_{1}>0 \text { y } 0 \text { en otro caso } \\
& y_{2}=1 \text { si } I_{2}>0 \text { y } 0 \text { en otro caso } \\
& I_{1}=\beta_{1} X_{1}+\varepsilon_{1} \\
& I_{2}=\beta_{2} X_{2}+\varepsilon_{2}
\end{aligned}
$$

además $E\left(\varepsilon_{1}\right)=E\left(\varepsilon_{2}\right)=0 ; V\left(\varepsilon_{1}\right)=V\left(\varepsilon_{2}\right)=1$ y $\operatorname{Cov}\left(\varepsilon_{1}, \varepsilon_{2}\right)=\rho$ ( no nulo).

Finalmente para estimar el modelo se supone que los errores siguen una normal bivariada.

En este artículo se considera que la variable Escolaridad admite los valores Estar en la escuela, que se denota 1, y No Estar, que es 0 . En el caso de la variable Trabajo, 1 significa estar en la fuerza de trabajo y 0 no hacerlo.

La variable Indice $I_{l}$ asociado a la escolaridad se interpreta como el beneficio neto de que el joven permanezca un año más en un establecimiento educacional, considerando como alternativa la no asistencia a la escuela ya sea para trabajar o para quedarse en el hogar. Si $I_{1}$ es positivo entonces es conveniente asistir. Si es negativo o cero, se escogerá no asistir a la escuela.

El segundo Indice $I_{2}$ mide el beneficio neto de trabajar para el mercado (denominado simplemente trabajar) considerando como alternativa no trabajar (trabajo para no mercado). Si este segundo índice es positivo, se elegirá entrar a la fuerza de trabajo.

En este contexto la decisión, por ejemplo, de que el joven continúe en la escuela y trabaje simultáneamente, lo que ocurre en un $2,1 \%$ de los casos, se expresa por $\left(y_{1}=1 ; y_{2}=1\right)$ y su probabilidad está determinada por la probabilidad de que $I_{1}>0$ y simultáneamente $I_{2}>0$.

Es interesante destacar que el modelo supone implícitamente que no hay complementariedad entre la decisión de estudiar o no estudiar y la decisión de trabajar o no trabajar, en el sentido que el beneficio neto de ambas es la suma de los beneficios netos de cada uno de ellas consideradas individualmente. 
También es conveniente puntualizar que el modelo considera que las decisiones son simultáneas y no secuenciales, como pudiera sugerir el hecho de haber colocado en primer término la variable escolaridad y luego la de trabajo.

La bibliografía presentada muestra la existencia de ciertas regularidades en las decisiones de deserción a la escuela y de entrada a la fuerza de trabajo. En la modelación de dichas decisiones se parte del supuesto que los padres consideran a los hijos como un factor de inversión que puede rendir beneficios tanto en el presente como en el futuro lejano, cuando ellos sean ancianos. Por esta razón, en la función de índice para la escolaridad se ha considerado que el beneficio de que este joven haga un año más de estudio tal como es percibido por la familia se puede asociar a: i) la percepción que tengan los padres del impacto de la educación formal en los ingresos futuros. Dicha percepción es mayor en padres con mayor educación formal y depende, además, del medio en que se desenvuelve la familia; ii) las posibilidades de sustitución del joven por otro miembro del hogar que se ha descrito por el número de personas, del hogar y por el hecho de que el joven sea el mayor de sus hermanos, y iii) por los rendimiento del joven como una medida de la probabilidad de éxito en lograr un grado más al cabo del año de estudio.

El costo de la decisión de mantener al joven en la escuela, por su parte, puede asociarse a la facilidad de acceso a, y las características de, los establecimientos educacionales. En este sentido, una mayor cantidad de establecimientos educacionales se puede asociar a más amplias posibilidades de elección y a la reducción de costos de acceso.

En este artículo la distinción de grados de accesibilidad a establecimientos educacionales se ha vinculado a zonas geográficas, distinguiéndose entre ubicación rural o tres diferentes tipos de ciudades agrupadas por su tamaño. También se ha agregado, como otra dimensión de la oferta, el porcentaje de colegios privados y subvencionados en la comuna. Se sabe que el tipo de colegio indica una diferencia de la calidad de los mismos. ${ }^{5}$

También se ha incluido el valor de uso del tiempo del menor en su casa, que se ha descrito por el ingreso per cápita del hogar, por el hecho de vivir con los padres, porque el hogar sea monoparental (mujer jefa de hogar) y por el número de hermanos menores de cinco años. Estas dos últimas variables se han incluido para destacar la situación de aquellos hogares en que el jefe de hogar debe preocuparse del cuidado de los niños y, simultáneamente, trabajar para el mercado con el objeto de proveer el ingreso necesario para el sustento de la familia. En estos hogares, el costo alternativo del niño como acompañante de sus hermanos menores aumenta con la edad, pues libera tiempo de la madre jefa de hogar.

Obviamente la agregación de los factores de beneficios con los de costo permite determinar los beneficios netos, que son los que en definitiva constituyen la función $I_{l}$.

Los beneficios netos de la decisión de trabajar se pueden asociar a las condiciones del mercado de trabajo para los jóvenes, que se han descrito por las

${ }^{5}$ Ver Sapelli y Vial $(2002,2003)$. 
variables Desempleo y Salario medio pertinentes y por los factores de beneficios netos de la decisión de continuar en la escuela. Las restantes variables no observadas que afectan a los beneficios netos de las decisiones de continuar en la escuela y de trabajar se han agrupado en $e_{1}$ y $e_{2}$ para las variables de decisión $I_{1}$ e $I_{2}$, respectivamente.

En resumen, se han considerado las siguientes variables:

- Variables de caracterización del niño como edad, sexo, ser hijo mayor y una medida de logro escolar. Estudios recientes han enfatizado el impacto de las características académicas de los jóvenes en la deserción (ver Eckstein y Wolpin, 1999). Se ha empleado la repitencia como variable de logro.

- Variables socioeconómicas del hogar, que incluyen ingresos de la familia, número de personas del hogar, hogares monoparentales, hermanos menores de seis años y el hecho de vivir con sus padres.

- Variables de percepción de los padres e impacto del medio ambiente en los beneficios de la educación, expresadas en términos de años de educación del padre y de la madre y otras variables que los vinculan a los otros hogares de la vecindad y que han sido construidas: la primera, como la diferencia entre los años de educación de cada padre y la educación promedio de los padres del entorno; la segunda, como la diferencia entre los años de educación de cada madre y la educación promedio de las madres del entorno, y la tercera, como la diferencia entre los ingresos de cada padre y el promedio de los ingresos de los padres del entorno. ${ }^{6}$

- Variables de oferta educacional dadas por: i) zona geográfica, para distinguir entre Santiago, las grandes ciudades, el resto de las ciudades y el área rural que es la variable de base, y ii) por las características del sistema educacional próximo, medido por la proporción de colegios según dependencia: municipalizado, particular subvencionado y particular pagado.

- Variables del mercado de trabajo que enfrentan los menores y que incluye el desempleo y el salario medio por comuna para el segmento de personas entre 14 y 19 años. ${ }^{7}$

Hipótesis de trabajo:

La construcción de las funciones $I_{1}$ e $I_{2}$ de beneficios netos determina un conjunto de hipótesis que surgen al considerar que cada uno de los factores de beneficios de las dos decisiones debieran asociarse positivamente con dichas decisiones, en tanto que los factores de costo debieran vincularse en forma nega-

${ }^{6}$ El entorno se ha medido por las personas que habitan en un segmento que es una unidad geográfica más pequeña que la comuna y, por lo tanto, en que las relaciones interpersonales son más intensas.

${ }^{7}$ Se ha considerado hasta los 19 años para el cálculo de las tasas de desempleo y de salario medio, porque las tasas son más robustas desde el punto de vista estadístico. 
tiva. Es así que pueden destacarse, como hipótesis a ser corroboradas, la relación positiva de la educación de los padres en la permanencia del menor en el sistema educacional y el efecto vecindario que tiende a contrarrestar el efecto específico y positivo de la educación de los padres en la permanencia en el sistema educacional del menor.

Otra hipótesis es el efecto positivo de la accesibilidad a los colegios y el efecto, también positivo, de la mayor disponibilidad de colegios privados y subvencionados. Entre los factores de costo puede considerarse el efecto negativo de la repitencia y el efecto negativo del mayor costo alternativo del tiempo del menor en el caso de hogares monoparentales con hermanos menores de cinco años. En la decisión de trabajar puede destacarse el efecto positivo del mayor salario medio de la comuna y el impacto negativo del desempleo. En lo que respecta al ingreso, la hipótesis de base es que aumentaría la asistencia escolar bajo el supuesto de que la educación es un bien normal. El impacto del ingreso del trabajo es ambiguo, porque puede predominar el efecto sustitución o el efecto ingreso.

Finalmente, parece conveniente verificar las hipótesis, que son parte de una tradición oral más que el resultado de consideraciones teóricas, sobre el tratamiento especial que tendrían los hijos varones (en el sentido que se les permitiría una deserción más tardía que a las hijas), o al hijo mayor (que tendrían que desertar antes para salir a trabajar y así contribuir a financiar los estudios de sus hermanos menores).

\section{Base de Datos y Análisis de los Resultados}

Los antecedentes empíricos se han obtenido de la encuesta CASEN de $1996 .^{8}$ En el Anexo se presenta un resumen de las diferentes variables consideradas.

Los resultados empíricos se presentan en el Cuadro 5 que entrega las estimaciones del modelo bivariado que se ha empleado. En primer término se analizará el impacto cualitativo de las variables a través de los signos de sus coeficientes y luego se calculan las elasticidades para disponer de una medida cuantitativa del efecto específico de cada una de ellas. Finalmente se comparan los resultados de este trabajo con los de la literatura.

\footnotetext{
${ }^{8}$ Las encuestas CASEN constituyen una base de datos que proporciona información sobre las características sociodemográficas de las personas (edad, sexo, educación, región, zona en que habita y condiciones de salud, entre otras), ingreso percibido y participación en el mercado laboral, categoría ocupacional y actividad económica en que se desempeña. La encuesta entrega un vasto conjunto de antecedentes de hogares, tiene representatividad nacional y regional y es suficientemente numerosa como para trabajar con diversas variables a la vez. Además, ha sido validada en numerosos trabajos anteriores.
} 
CUADRO 5

LAS DECISIONES DE DESERTAR Y DE INGRESAR AL MERCADO DEL TRABAJO

\begin{tabular}{|c|c|c|}
\hline & $\begin{array}{c}(1) \\
\text { Asiste a establecimiento } \\
\text { educacional }\end{array}$ & $\begin{array}{c}\text { (2) } \\
\text { Tiene empleo o } \\
\text { busca trabajo }\end{array}$ \\
\hline Repitencia & $\begin{array}{c}-0.357 \\
(15.06)^{* *}\end{array}$ & $\begin{array}{c}0.197 \\
(10.64)^{* *}\end{array}$ \\
\hline Educación del padre & $\begin{array}{c}0.068 \\
(3.27)^{* *}\end{array}$ & $\begin{array}{l}-0.067 \\
(3.09)^{* *}\end{array}$ \\
\hline Educación de la madre & $\begin{array}{c}0.069 \\
(2.94)^{* *}\end{array}$ & $\begin{array}{r}0.007 \\
(0.27)\end{array}$ \\
\hline Hermanos entre 0 y 5 años & $\begin{array}{l}-0.229 \\
(3.58)^{* *}\end{array}$ & $\begin{array}{l}0.008 \\
(0.12)\end{array}$ \\
\hline Hijo mayor & $\begin{array}{r}-0.066 \\
(0.83)\end{array}$ & $\begin{array}{l}0.252 \\
(3.10)^{* *}\end{array}$ \\
\hline Mujer jefa de familia & $\begin{array}{l}-0.522 \\
(2.74)^{* *}\end{array}$ & $\begin{array}{l}0.011 \\
(0.06)\end{array}$ \\
\hline Número personas en el hogar & $\begin{array}{l}0.025 \\
(0.97)\end{array}$ & $\begin{array}{l}0.092 \\
(4.01)^{* *}\end{array}$ \\
\hline Vive con sus padres & $\begin{array}{c}0.719 \\
(6.62)^{* *}\end{array}$ & $\begin{array}{r}-0.253 \\
(1.93)\end{array}$ \\
\hline Gran Santiago & $\begin{array}{r}-0.112 \\
(0.98)\end{array}$ & $\begin{array}{r}-0.102 \\
(0.88)\end{array}$ \\
\hline Otras grandes ciudades & $\begin{array}{l}0.170 \\
(1.09)\end{array}$ & $\begin{array}{l}0.059 \\
(0.40)\end{array}$ \\
\hline Resto de ciudades & $\begin{array}{l}0.198 \\
(2.31)^{*}\end{array}$ & $\begin{array}{r}-0.070 \\
(0.77)\end{array}$ \\
\hline Ingreso per cápita & $\begin{array}{c}0.000 \\
(2.58)^{* *}\end{array}$ & $\begin{array}{l}0.000 \\
(0.50)\end{array}$ \\
\hline Ingreso per cápita al cuadrado & $\begin{array}{l}-0.000 \\
(2.04)^{*}\end{array}$ & $\begin{array}{l}0.000 \\
(0.05)\end{array}$ \\
\hline Sexo & $\begin{array}{c}0.099 \\
(1.46)\end{array}$ & $\begin{array}{l}0.240 \\
(3.12)^{* *}\end{array}$ \\
\hline Diferencia de ingreso per cápita & $\begin{array}{r}-0.000 \\
(1.27)\end{array}$ & $\begin{array}{r}-0.000 \\
(0.04)\end{array}$ \\
\hline Diferencia de educación del padre & $\begin{array}{l}-0.050 \\
(2.08)^{*}\end{array}$ & $\begin{array}{l}0.068 \\
(2.92)^{* *}\end{array}$ \\
\hline Diferencia de educación de la madre & $\begin{array}{r}-0.019 \\
(0.78)\end{array}$ & $\begin{array}{r}-0.028 \\
(1.13)\end{array}$ \\
\hline Proporción colegios particulares & $\begin{array}{l}0.275 \\
(0.54)\end{array}$ & $\begin{array}{r}-0.097 \\
(0.22)\end{array}$ \\
\hline Proporción colegios subvencionados & $\begin{array}{r}0.248 \\
(1.14)\end{array}$ & $\begin{array}{l}0.046 \\
(0.23)\end{array}$ \\
\hline Edad 15 años & $\begin{array}{l}-0.449 \\
(3.75)^{* *}\end{array}$ & $\begin{array}{c}0.255 \\
(2.27)^{*}\end{array}$ \\
\hline Edad 16 años & $\begin{array}{l}-0.653 \\
(5.79)^{* *}\end{array}$ & $\begin{array}{l}0.442 \\
(4.28)^{* *}\end{array}$ \\
\hline Edad 17 años & $\begin{array}{l}-0.761 \\
(6.64)^{* *}\end{array}$ & $\begin{array}{l}0.669 \\
(5.93)^{* *}\end{array}$ \\
\hline Salario medio en comuna & & $\begin{array}{l}0.000 \\
(0.35)\end{array}$ \\
\hline Tasa desempleo 14-19 años en comuna & & $\begin{array}{r}0.150 \\
(0.75)\end{array}$ \\
\hline Constante & $\begin{array}{l}0.007 \\
(0.03)\end{array}$ & $\begin{array}{l}-2.079 \\
(9.38)^{* *}\end{array}$ \\
\hline Observaciones & 7066 & 7066 \\
\hline
\end{tabular}

Estadísticas robustas, valor de razón $\mathrm{Z}$ en paréntesis

$*$ significativa al $5 \%$; $*$ significativa al $1 \%$ 


\subsection{Análisis Cualitativo de los Resultados del Modelo}

Edad del niño: La edad incide negativamente en la probabilidad de asistir y positivamente en la de trabajar. En ambos casos los coeficientes son significativos. Además, los coeficientes de las diferentes edades, que se han introducido a través de variables dicotómicas aumentan en términos absolutos al considerarse edades mayores. Esto puede asociarse con un impacto de la edad no lineal con el paso del tiempo, lo que sería consistente con la ideas de un aumento del costo alternativo del joven tanto en su actividad dentro del hogar como en el mercado de trabajo. De manera que a mayor edad existe una mayor dimensión de "espejo" entre ambas decisiones.

Repitencia: A mayor repitencia, menor la asistencia y mayor la probabilidad de trabajar; los coeficientes, en términos absolutos, son significativamente diferentes entre ellos, por lo cual se nota que quienes se retiran por repetición no necesariamente se van a trabajar.

Educación del padre: Con relación a esta variable se nota efectivamente un efecto espejo en el sentido que la educación del padre contribuye a aumentar la asistencia y a disminuir el trabajo en muy similares proporciones.

Educación de la madre: En este caso no se da el efecto espejo. La educación de la madre contribuye a aumentar la probabilidad de asistencia, pero no tiene incidencia significativa respecto a la probabilidad de trabajar.

Diferencia entre la educación de cada padre y las medias del segmento de residencia: La diferencia positiva entre la educación del padre y la educación promedio de los padres de la zona pertinente (segmento) contribuye a aumentar la probabilidad que una persona trabaje y a disminuir la probabilidad de asistencia al colegio. Curiosamente, la variable que evalúa esa diferencia para la madre no tiene efecto significativo. Como estas variables están definidas como la educación de cada padre menos la del segmento, el hecho de que su coeficiente para el padre diera positivo en el caso de la decisión de trabajar indica que si la educación del padre es menor a la media, cuanto menor a la media es, menos probable es que el hijo trabaje. Este "efecto vecindario" también se observa en el caso de decisión de asistencia. Un padre con educación menor al promedio del segmento, cuanto menor es, mayor es la probabilidad que el hijo asista. De manera que en ambos casos el efecto actúa para moderar la tendencia de que a menor educación del padre, más se trabaja y más se deserta. Las personas que viven en un vecindario con niveles de educación diferentes a los de ellos tienden a asimilarse al comportamiento del vecindario más que al comportamiento de otras personas con similar educación.

Un punto importante es el impacto significativo de la educación del padre en ambas decisiones: la de trabajo y la de asistencia (tanto por el nivel como por la diferencia), en tanto que el impacto de la madre se observa en asistencia solamente. Por otra parte, el impacto de ambos sobre la decisión de asistir, medidos por la magnitud de los coeficientes, serían similares. En el caso de Chile, la apreciación 
tradicional respecto a la importancia sólo de la madre no es respaldada por los datos. Como se estableció anteriormente, este resultado también se obtiene en otros países, como Ghana, con una metodología similar a la usada en este trabajo.

Número de hermanos menores de cinco años: Tener muchos hermanos pequeños contribuye a disminuir la probabilidad de asistencia, pero no aumenta la probabilidad de trabajar, con lo cual se demuestra que en el caso de hermanos pequeños es probable que la conducta principal sea desertar para quedarse a cuidar a sus hermanos.

Hermano mayor: Ser el mayor contribuye a aumentar la probabilidad de que trabaje, pero no que deje de asistir. Es importante el resultado de que ser mayor no afecta la asistencia, puesto que muestra que los datos no avalan la idea del tratamiento preferencial en lo que respecta a educación de los hijos mayores.

Jefe de familia de sexo femenino: Que el jefe de familia sea mujer disminuye la probabilidad de asistencia, pero no afecta la probabilidad que trabaje, por lo cual es probable que los hijos se queden a cuidar la casa. Este resultado, al igual que el de hermanos menores muestra que, en ciertos hogares, la disponibilidad de alternativas para cuidar al resto de la familia podrían aumentar la asistencia.

Número de personas en el núcleo: Cuanto mayor el número de personas en el núcleo, mayor la probabilidad de que el niño trabaje, pero dicho número no afecta la probabilidad de asistencia. O sea, esta variable opera en forma similar a ser el hermano mayor.

El niño vive con sus padres: El vivir con los padres tiene un efecto positivo sobre la probabilidad de asistir, y un efecto negativo sobre la de trabajar. En principio, podría no ser claro si esto es un efecto altruista o una indicación de un efecto ingreso (vive con ambos padres por oposición a vivir solo); sin embargo, dado todo lo que se controla en la regresión podría decirse que hay un efecto altruista (alternativamente puede pensarse que hay mayor probabilidad de recibir dinero del niño en el futuro).

Localización (donde las opciones son: Santiago, grandes ciudades, otros centros urbanos y sector rural): En general estas variables dicotómicas no son significativas, lo cual puede ser inesperado. Sin embargo, dicho comportamiento es esperable si se considera que los factores de diferencia rural/urbano, están incorporados en otras variables como, por ejemplo, vecindario, participación en colegios particulares subvencionados o particulares pagados y tamaño del hogar.

Ingreso per cápita del hogar: Un mayor ingreso aumenta la probabilidad de asistir, pero no influye sobre la probabilidad de trabajar. La incorporación de una variable cuadrática indica la existencia de no linealidades, que se expresa porque su efecto va disminuyendo a medida que aumenta el ingreso.

Sexo del niño: Todo lo demás igual, los hombres tienen una probabilidad mayor de trabajar. Sin embargo, el ser hombre no influye sobre la probabilidad de 
asistencia. El hecho de que una variable influya sobre una decisión, pero no sobre la otra, ya se había detectado en el caso de las variables "hermano mayor" y "número de personas en el hogar". 9 Lo interesante de este último resultado es que los niños varones asisten menos. Entonces, el hecho de que la variable género del niño no fuera significativa quiere decir que esa diferencia es totalmente explicada por el modelo, a través de otras variables. Podría ser, por ejemplo, que los hombres tuvieran mayor repitencia (peor performance en el sistema educativo) y que al controlar por esta variable se elimina la diferencia de sexos. ${ }^{10}$

Diferencia del ingreso per cápita con la media del segmento: Una vez que se controla por la diferencia de educación entre los padres del niño y el resto de la comunidad, las diferencias de ingreso no importan y no influyen ni en la probabilidad de asistir ni en la probabilidad de trabajar.

Porcentaje de colegios particulares pagados, particulares subvencionados y municipalizados: Esta variable no es significativa, de manera que la estructura de la oferta no parece afectar ni la decisión de asistir ni la de trabajar.

Las variables que caracterizan el mercado laboral de la comuna no resultaron significativas. Puede contribuir a explicar esta situación el hecho que la economía venía de un período de expansión a tasas muy altas.

Finalmente, debe destacarse la existencia de una correlación negativa entre los no observables que contribuyen a la decisión de asistir y a la de trabajar, confirmando que entre lo no observables también existe un efecto espejo similar al que se da para algunos observables. O sea, en términos de no observables, si hay una probabilidad alta de asistir esta se correlaciona con una probabilidad baja de trabajar y viceversa.

Elasticidades: Para comparar los efectos de las variables es importante calcular las elasticidades, ya que la dimensión de su efecto marginal no puede deducirse del tamaño de los coeficientes. Se presentan las estimaciones relacionadas con las variables más importantes. Sin embargo, la edad, que tiene un efecto muy importante sobre ambas decisiones, y en particular la de trabajar, ha debido excluirse, puesto que fue incorporada a través de variables diferentes.

De los resultados presentados en el Cuadro 6 destaca la elasticidad de la educación del padre en la decisión de trabajar. En la decisión de estudiar destaca la similitud de los efectos de la educación del padre y de la madre, aunque en términos absolutos su magnitud es mucho menor al efecto de la educación del padre sobre la decisión de trabajar. El ingreso per cápita tiene un efecto pequeño en relación a estas variables, y su efecto es sólo importante en la decisión de asistir y no en la de trabajar. La medida de logro utilizada (una estimación de la repitencia) tiene también un efecto importante en ambas decisiones. Quien repite es más probable que abandone y más probable que trabaje.

9 Estas variables muestran que la metáfora del "espejo" tiene limitaciones como descripción del comportamiento en esta área.

10 Se agradecen los comentarios de un árbitro anónimo respecto de este punto. 


\begin{tabular}{lcc}
\multicolumn{3}{c}{$\begin{array}{c}\text { CUADRO 6 } \\
\text { ELASTICIDADES }\end{array}$} \\
\hline Variables & $\begin{array}{c}\text { Probabilidad de } \\
\text { asistir }\end{array}$ & $\begin{array}{c}\text { Probabilidad de } \\
\text { trabajar }\end{array}$ \\
\hline Repitencia & $-0,012$ & 0,19 \\
Educación padre & 0,040 & $-1,31$ \\
Educación madre & 0,038 & 0,00 \\
Ingreso per cápita & 0,016 & 0,00 \\
Diferencia de educación del padre & $-0,001$ & 0,04 \\
\hline
\end{tabular}

\subsection{Comparación con Otros Resultados}

El Cuadro 7 entrega un resumen del efecto de las principales variables incluidas en el modelo. Su objetivo consiste en facilitar las comparaciones con los resultados de otros trabajos presentados en el Cuadro 1.

\section{CUADRO 7 \\ RESUMEN DEL IMPACTO DE LAS PRINCIPALES VARIABLES CONSIDERADAS}

\begin{tabular}{lc}
\hline Variable & Impacto en asistencia \\
\hline Gasto/ingreso & Positivo y pequeño \\
Edad & Negativo en asistencia y positivo en trabajo \\
Sexo (mujer) & No significativo \\
Educación del padre & Positivo en asistencia y negativo en trabajo \\
Educación de la madre & Positivo en asistencia \\
Variables demográficas familia & Importantes \\
Correlación de no observables con & Negativa \\
la decisión de trabajar & \\
\hline
\end{tabular}

La comparación de los resultados con los que entrega la literatura internacional (ver Cuadro 1) permite algunas conclusiones interesantes. Comentaremos primero los resultados en que ha habido discrepancia en la literatura. Nos referimos básicamente al efecto del ingreso y del sexo del niño sobre la probabilidad de asistir a la escuela.

En primer lugar encontramos, como en varios trabajos en la literatura, que el efecto del ingreso sobre la decisión de asistencia es pequeño. Este punto contrasta con el resultado de Beyer para Chile y es coincidente con los dos otros trabajos que usan similar metodología a la nuestra, mostrando que quizás el resultado de que el ingreso es importante es sensible a la metodología de estimación. El Gráfico 1 muestra que el impacto de un cambio del ingreso de 20.000 mensuales per 
cápita a 300.000 tiene un efecto de incrementar la probabilidad de asistencia en sólo 2,8 puntos porcentuales.

El sexo del niño no es importante en la decisión de asistir, a diferencia de varios países africanos y de Perú, y en coincidencia con el resultado de Beyer para Chile.

Ya se ha destacado el resultado interesante respecto a la educación del padre y de la madre. Ambos son significativos para la decisión de asistir, y concuerdan con los obtenidos por Canagarajah y Coulombe para Ghana.

Por otra parte, las características del hogar resultan importantes en este trabajo, en forma coincidente también con los resultados obtenidos por Canagarajah y Coulombe para Ghana, y en contraposición con el estudio de Ranjan para Pakistán.

Finalmente, la correlación entre los no observables resulta significativa y negativa, lo que concuerda con los otros dos trabajos con similar metodología a la nuestra.

\section{CONCLUSIONES}

La primera conclusión del artículo es que efectivamente es necesario analizar conjuntamente la decisión de estudiar, o desertar, y la de trabajar, puesto que la correlación entre los no observables en ambas decisiones es significativamente diferente de cero.

Los resultados encontrados son en general consistentes con los de otros trabajos que utilizan la misma metodología. Entre ellos cabe destacar que el ingreso no es una variable tan importante como determinante de la decisión de trabajar como suele creerse. Segundo, que la educación del padre parece mucho más importante de lo usualmente considerado, y ello aparece realzado al estudiar las decisiones de desertar y de trabajar conjuntamente. Tercero, que no hay discriminación contra la mujer ni contra el mayor a la hora de decidir la asistencia a educación media. Sin embargo, sí es cierto que la probabilidad de trabajar es mayor en los hombres y aumenta con la edad.

Además, una vez que se controla por las variables utilizadas en nuestro estudio, la localización no resulta relevante, demostrando que las decisiones en el sector rural y en el sector urbano se toman según similares parámetros.

La estructura de la oferta no parece afectar la decisión de asistir, pero sí lo hace con la de trabajar. Este tema deberá estudiarse con más detalle en el futuro, si se puede obtener más información sobre las escuelas.

Finalmente, se descubre un interesante "efecto vecindario", que lleva a personas con educación diferente a la de su vecindario se comporten en forma similar a ellos. Por ejemplo, la influencia de la madre sobre la decisión de asistir es mayor para madres de baja educación que viven en un vecindario más educado, en que los niños van con mayor probabilidad a la educación media. Un efecto similar existe respecto a la diferencia de la educación del padre y la decisión de trabajar. 
Una conclusión importante es que si bien en algunos aspectos ambas decisiones sí son dos caras de un mismo proceso de decisión, pensar que los jóvenes desertan para emplearse en un trabajo es simplificar la decisión, ya que muchos jóvenes desertan y se quedan en el hogar.
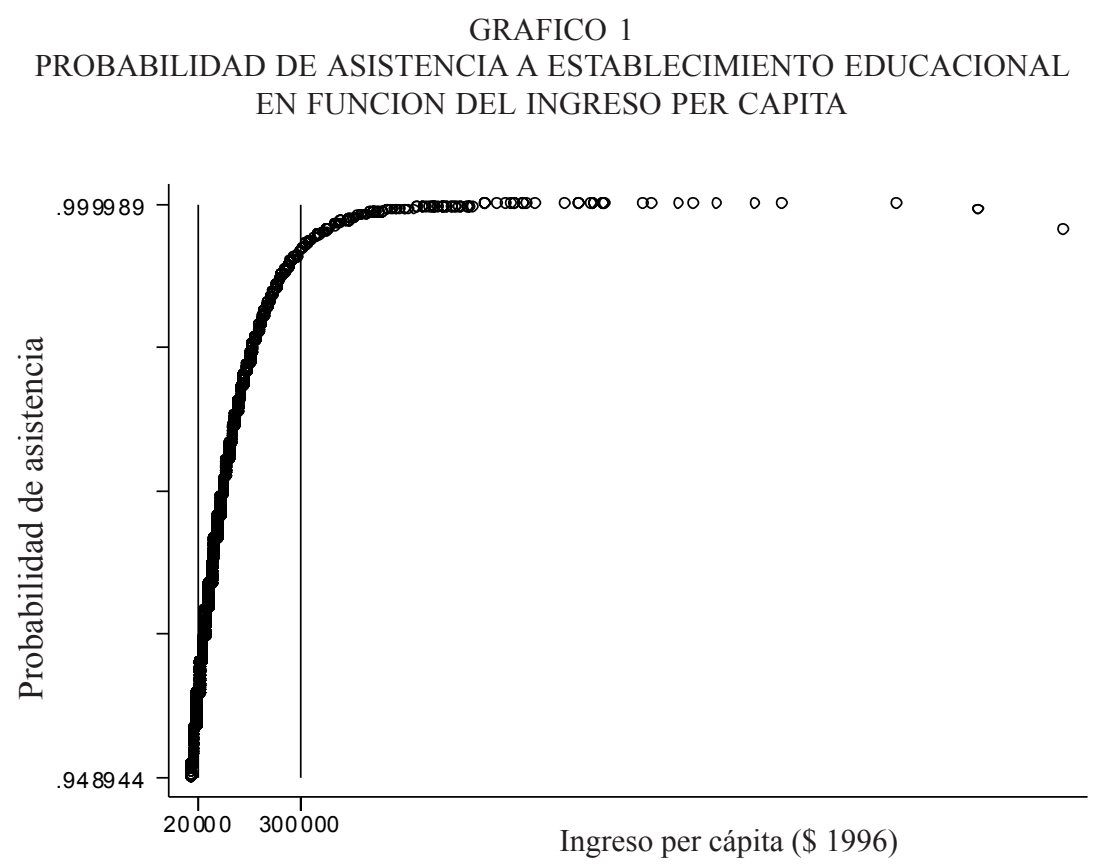

Con relación a las conclusiones de política, parece importante destacar que, dado que el ingreso no cumple un papel importante, la opción de dar un subsidio en dinero para asistir no parece ser la más adecuada. En efecto, el Gráfico 1 muestra que la probabilidad de asistir es bastante alta y, además, el efecto del ingreso en el cambio de dicha probabilidad es muy bajo. En efecto, un subsidio del $10 \%$ del ingreso para una nivel de ingreso per cápita de $\$ 17.000$, que en 1996 correspondía al nivel de corte de la indigencia, incrementaría la probabilidad de asistencia en $0,35 \%$ puntos porcentuales (de $76,5 \%$ a $76,85 \%$ ). Un subsidio de $20 \%$ del ingreso, incrementaría la probabilidad de asistencia en 0,7 puntos porcentuales (de 76,5\% a 77,21\%). Por otra parte, este es un programa difícil de focalizar, lo que redunda en un alto costo por joven que efectivamente cambia su decisión de asistir debido al subsidio.

Finalmente, sería interesante en el futuro conectar estas decisiones con resultados como los de Sapelli (2003), que demuestran que las tasas de retorno de la educación media son bajas durante los primeros tres años y que los retornos de la experiencia laboral son razonablemente altos. Sólo para quienes esperan graduarse de media y en particular para quienes pretenden seguir a la universidad es 
claramente rentable continuar en la educación media. Esto podría explicar por qué sin montos grandes de ingreso no puede cambiarse la decisión de desertar.

Una avenida más promisoria se asocia con el diseño de mecanismos para apoyar a las familias en el cuidado de los menores y, en particular, en la provisión de educación preescolar. En efecto, la existencia de hermanos menores en el hogar reduce fuertemente la probabilidad de asistencia de los mayores a la escuela. Es así que el primer hermano menor de cinco años incrementa la deserción en seis puntos porcentuales; el segundo en otros seis puntos porcentuales adicionales y el tercero en nueve puntos porcentuales más. Por otra parte, un programa de este tipo puede focalizarse con mayor facilidad. Finalmente, la educación preescolar tiene muy altos retornos al aumentar el capital humano de los menores que se benefician de ella (ver Heckman y Carneiro, 2003).

\section{REFERENCIAS}

Angrist J. y W. Evans (1998), "Children and Their Parents Labor Supply: Evidence from Exogenous Variation in Family Size", American Economic Review 88: 450-477.

Basu, K. y P. H. Van (1998), “The Economics of Child Labor”, American Economic Review $88: 412-427$.

Basu, K. y P. H. Van (1999), “The Economics of Child Labor: Replay”, American Economic Review 89: 1386-1388.

Beyer, H. (1998), “¿Desempleo juvenil o un problema de deserción escolar?”, Serie Documentos de Trabajo $\mathrm{N}^{\circ} 277$, Centro de Estudios Públicos.

Bravo, D. y Contreras, D. (2000), "Salario Mínimo y Deserción Escolar: revisión de la evidencia empírica para Chile". Departamento de Economía, Universidad de Chile, mimeo.

Canagarajah, S. y Coulombe, H. (1998), "Child Labor and Schooling in Ghana", Policy Research Working Paper 1844. The World Bank: Washington DC.

Card, D. y Lemieux, T. (1997), “Adapting to circumstances: the evolution of work, school and living arrangements among NorthAmerican youths", National Bureau of Economic Research, Working Paper 6142.

Card, D. y Lemieux, T. (2000), "Dropout and enrollment trends in the post-war period: what went wrong in the 1970's", National Bureau of Economic Research, Working Paper 7658.

Coloma, F. y B. Vial (2003), "Desempleo e Inactividad Juvenil en Chile", Cuadernos de Economía 119: 149-171.

Cooper, A. (1999), “Elección de Colegios y Demanda por Atributos”, Seminario de Título, Instituto de Economía, Pontificia Universidad Católica de Chile.

Eckstein, Z. y K. Wolpin (1999), "Why Youths drop out of High School: the Impact of Preferences, Opportunities, and Abilities", Econometrica 67.

Gertler, P. y Glewwe, P. (1992), "The Willingness to Pay for Education for Daughters in Contrast to Sons: Evidence from Rural Perú", The World Bank Economic Research, 6: 171-188.

Greene, W. (2000), Econometric Analysis, Fourth Edition. Prentice Hall, New Jersey.

Heckman, J. y P. Carneiro (2003), “Human Capital Policy”, NBER Working Papers 9495. 
Kane, T. J. (1994), “College Entry by Blacks since 1970: the Role of College Costs, Family Background, and the Returns to Education", Journal of Political Economy, 102: 878-911.

Margo, R. yT. A. Finegan (1993), “The Decline in BlackTeenage Labor-force Participation in the South, 1900-1970: the Role of Schooling",American Economic Review, 83: 234-247.

Neumark, D. yW. Wascher (1995), "Minimum-wage Effects on School and Work Transitions of Teenagers", American Economic Review, 85: 244-249.

Paredes, R. y R. Sanhueza (1996), "Minimum Wages and School Dropouts in Chile", mimeo, Universidad de Chile.

Ranjan, R. (2000), "How Child Labor and Child Schooling Interact with Adult Labor", World Bank Policy Research Working Paper 2179.

Ravallion, M. y Q. Wodon (1999), "Does Child Labor Displace Schooling? Evidence on Behavioral Responses to an Enrollment Subsidy", World Bank Policy Research Working Paper 2116.

Ravallion, M. y Q. Wodon (2000), “Does Child Labor Displace Schooling?”, Economic Journal 110 (462): C158-C176.

Sapelli, C. (2003), "Ecuaciones de Mincer y las Tasas de Retorno a la Educación en Chile: 1990-1998", Documento de Trabajo No 254, Instituto de Economía, Pontificia Universidad Católica de Chile.

Sapelli, C. y B. Vial (2002), "The Performance of Private and Public Voucher Schools in Chile", Cuadernos de Economía 118: 423-454.

Sapelli, C. y B. Vial (2003), "Peer Effects and the Relative Performance of Voucher Schools in Chile", Documento de Trabajo N ${ }^{\circ}$ 256, Instituto de Economía, Pontificia Universidad Católica de Chile.

Skoufias, E. y S. W. Parker (2001), "Conditional Cash Transfers and the Impact on Child Work and Schooling: Evidence from the PROGRESAProgram in Mexico", Economia 2: 45-96.

Skyt N. H. (1998), "Child Labor and School Attendance: two Joint Decisions", Center for Labor Market and Social Research, University of Aarhus, Denmark.

Swinnerton, K.A. y C.A. Rogers (1999), "The Economics of Child Labor: Comment", American Economic Review 89: 1382-1385.

Willis, R. J. y S. Rosen (1979), “Education and Self-Selection”, Journal of Political Economy, $87,5:$ S7-36. 


\section{ANEXO}

En el CuadroA1 puede observarse que poco menos del $90 \%$ de los jóvenes de entre 14 y 17 años está en la escuela, en tanto que un 7\% reporta estar trabajando. El resto lo constituyen jóvenes que se encuentran inactivos, es decir, trabajando para el hogar (no mercado). Todas esta personas inactivas podrían estar trabajando o estudiando, puesto que, como se dijo anteriormente, se han eliminado aquellas personas que están fuera del ciclo de estudio por haberlo terminado, o las que están inhabilitadas para hacerlo (enfermedades, servicio militar etc.)

CUADRO A1

DESCRIPCION DE LAS VARIABLES CONSIDERADAS

\begin{tabular}{|c|c|c|c|c|}
\hline Variable & Media & $\begin{array}{l}\text { Desviación } \\
\text { estándar }\end{array}$ & Mínimo & Máximo \\
\hline Asiste a la escuela & 0,897 & 0,303 & 0 & 1 \\
\hline Trabaja & 0,066 & 0,249 & 0 & 1 \\
\hline Repitencia & 0,384 & 1,592 & -2 & 11 \\
\hline Educación del padre & 9,674 & 4,310 & 1 & 22 \\
\hline Educación de la madre & 9,186 & 3,910 & 0 & 21 \\
\hline Hermanos entre 0 y 5 años & 0,261 & 0,513 & 0 & 4 \\
\hline Hijo mayor & 0,458 & 0,498 & 0 & 1 \\
\hline Mujer jefa de familia & 0,031 & 0,173 & 0 & 1 \\
\hline Número personas en el hogar & 5,032 & 1,313 & 2 & 12 \\
\hline Vive con sus padres & 0,940 & 0,238 & 0 & 1 \\
\hline Gran Santiago & 0,298 & 0,457 & 0 & 1 \\
\hline Otras grandes ciudades & 0,101 & 0,301 & 0 & 1 \\
\hline Resto de ciudades & 0,440 & 0,496 & 0 & 1 \\
\hline Ingreso per cápita & $8,54 \mathrm{E}+4$ & $1,40 \mathrm{E}+5$ & 0 & $2,41 \mathrm{E}+6$ \\
\hline Ingreso per cápita al cuadrado & $2,61 \mathrm{E}+10$ & $1,62 \mathrm{E}+11$ & 0 & $5,83 \mathrm{E}+12$ \\
\hline Sexo & 0,501 & 0,500 & 0 & 1 \\
\hline Diferencia de ingreso per cápita & $-1,66 \mathrm{E}+4$ & $1,16 \mathrm{E}+5$ & $-2,34 \mathrm{E}+6$ & $1,91 \mathrm{E}+6$ \\
\hline $\begin{array}{l}\text { Diferencia de educación del } \\
\text { padre }\end{array}$ & 0,242 & 2,900 & $-9,8$ & 13,2 \\
\hline $\begin{array}{l}\text { Diferencia de educación. de la } \\
\text { madre }\end{array}$ & 0,184 & 2,850 & $-11,7$ & 12 \\
\hline Proporción colegios particulares & 0,113 & 0,131 & 0 & 0,7 \\
\hline $\begin{array}{l}\text { Proporción colegios } \\
\text { subvencionados }\end{array}$ & 0,359 & 0,157 & 0 & 1 \\
\hline Edad 14 años & 0,246 & 0,431 & 0 & 1 \\
\hline Edad 15 años & 0,264 & 0,441 & 0 & 1 \\
\hline Edad 16 años & 0,259 & 0,438 & 0 & 1 \\
\hline Edad 17 años & 0,231 & 0,421 & 0 & 1 \\
\hline Salario medio en comuna & 437,8 & 304,6 & 68,1 & 2500 \\
\hline $\begin{array}{l}\text { Tasa desempleo } 14-19 \text { años en } \\
\text { comuna }\end{array}$ & 0,184 & 0,148 & 0 & 1 \\
\hline
\end{tabular}

Fuente: Cálculo de los autores sobre información de encuesta CASEN 1996.

El Cuadro A1 muestra, además, que un 38\% de los jóvenes presenta problemas de repitencia. Esta variable es indicativa de problemas académicos que pueden estimular la deserción escolar. Por otra parte, se observa que los padres reportan 9,7 años de educación en promedio, en tanto que las madres 9,2 años, lo que, tiende a confirmar, en este segmento, la homogeneidad educacional entre 
hombres y mujeres en Chile. Un 26\% reporta tener hermanos menores de hasta cinco años, situación que puede constituir un obstáculo para la continuación en la escuela por la necesidad de cuidado de dichos niños. Esta necesidad de cuidado se torna más acuciante en el caso de hogares en que el jefe de hogar es mujer, puesto que muchos de esos hogares son monoparentales y, por lo tanto, la jefa de hogar debe trabajar para el mercado. En este grupo, el número de hogares con jefe de hogar femenino es pequeño en relación al total nacional: alcanza a sólo un 3\%.

Un $45 \%$ de los jóvenes reporta ser el hijo mayor. Este porcentaje puede parecer alto, pero debe tenerse presente que la población en estudio está constituida sólo por los jóvenes entre 14 y 17 años que son de los mayores de la casa toda vez que los de más edad habrían salido del hogar o se habrían casado. Es interesante recordar que las cifras de este estudio se remontan a 1996. Este hecho ayuda a entender, además, el alto número de personas por hogar, que alcanza a cinco, conjuntamente con el hecho que se está considerando un conjunto de hogares en que la familia estaría en su máximo nivel de tamaño, toda vez que los hogares incluidos son sólo aquellos en que hay jóvenes entre 14 y 17 años. Por otra parte, un alto porcentaje de jóvenes, que alcanza el 94\%, declara que vive con sus padres.

Al considerar la localización de estos jóvenes puede observarse que un $30 \%$ vive en Santiago, un $10 \%$ en las otras dos grandes ciudades, un $44 \%$ en otras ciudades y pueblos y el 16\% restante en el campo. Es interesante destacar que Santiago agrupa un porcentaje menor de jóvenes en relación a su participación en la población total, que es alrededor del $38 \%$. También es interesante destacar el alto porcentaje de menores que se ubica en el resto urbano, que en general ofrece menos posibilidades de elección en términos educacionales. En Santiago, un 45\% de los menores asiste a colegios particulares subvencionados y un $15 \%$ lo hace en colegios particulares, en tanto que en el resto urbano un 30\% va a colegios particulares subvencionados y sólo un $7 \%$ a particulares.

El ingreso per cápita promedio de los hogares considerados alcanza a $\$ 85.000$ y es un $9 \%$ superior al ingreso per cápita promedio nacional.

Las siguientes cinco variables muestran la relación de cada familia con el medio en que se ubica. La primera indica la relación entre el ingreso per cápita del hogar y el ingreso también per cápita promedio de la zona en que se ubica la familia. La segunda, la diferencia entre la escolaridad del padre y la escolaridad promedio de los padres de la zona. La tercera indica la diferencia entre la escolaridad de la madre y el promedio de escolaridad de las madres de la zona. Como zona se ha considerado el segmento que es una unidad geográfica más pequeña que la comuna. Estas variables se han introducido para establecer el impacto del ambiente en las decisiones de cada uno de las familias consideradas. Finalmente se han examinado dos variables que miden la proporción de alumnos en colegios particulares subvencionados y particulares pagados, que pueden ser consideradas proxies de disponibilidad de diferentes tipos de colegios y por la otra de preferencias de los padres de la zona, expresadas por la proporción de jóvenes que asiste a los diferentes tipos de establecimientos educacionales. En este caso se desea estudiar en 
qué medida dichas características de la zona en que habita la familia afectan su comportamiento. Las medias de estas diferencias no son nulas debido a que los cálculos de los promedios del segmento se han obtenido agregadamente y, por lo tanto, los valores inexistentes (missing values) para cada una de las variables se han extendido a todas las otras.

La edad de los jóvenes se ha incorporado a través de cuatro variables dicotómicas, con el objeto de tomar en cuenta posibles no linealidades del efecto de la edad en las decisiones de estudiar o trabajar. Es interesante destacar que la proporción de jóvenes de las diferentes edades es muy similar, con alrededor de un $25 \%$ en cada una de ellas.

Finalmente, se han introducido dos variables para describir otras tantas características del mercado laboral: salario promedio y tasa de desempleo ambas para el grupo de edad pertinente. Estas variables tienen por objeto determinar el efecto de las condiciones del mercado laboral en las decisiones de desertar y, obviamente, en las de trabajar para el mercado. Es interesante destacar que el salario está medido en pesos por hora y que el desempleo promedio para la población de jóvenes, que alcanza al 18,4\%, es casi el triple del desempleo nacional que en ese año totalizaba un $6,5 \%$. 
Deserción Escolar y Trabajo JuVenil 